\title{
On the positioning error of a 2-DOF spherical parallel wrist with flexible links and joints - an FEM approach
}

\author{
G. Palmieri \\ Università degli Studi eCampus, Via Isimbardi 10, 22060 Novedrate (CO), Italy \\ Correspondence to: G. Palmieri (giacomo.palmieri@uniecampus.it)
}

Received: 21 October 2014 - Revised: 7 January 2015 - Accepted: 10 February 2015 - Published: 26 February 2015

\begin{abstract}
This paper deals with an elasto-static analysis of a 2-DOF (degrees of freedom) spherical parallel wrist where the links and the joints are considered flexible. Theoretically, the mobile platform of the wrist rotates around a fixed point which is the intersection of all the joint axes. However, if the flexibility of the limbs is taken into account, while the base platform (BP) and the mobile platform (MP) are assumed rigid, two centers can be identified: one for the BP and the other for the MP. In general such points are not coincident; as a result, the positioning of the MP is affected by inaccuracies, which can be evaluated in terms of displacement and orientation errors. A finite-element method (FEM) approach is used to analyze the problem in a series of configurations of the wrist; the results are elaborated in order to obtain continuous maps of the errors over the workspace of the machine.
\end{abstract}

\section{Introduction}

A spherical parallel wrist is a manipulator formed by at least two kinematic chains (limbs) connecting a base platform (BP) to a mobile platform (MP) which is characterized by a spherical motion with 2 or 3 degrees of freedom (DOFs). Furthermore, all the links of the kinematic chains can have only a spherical motion; thus, in spherical parallel manipulators with revolute joints, each limb is composed of links connected by revolute joints whose axes intersect at a common point which represents the center of the spherical motion. Several examples of 3-DOF spherical parallel wrists used to orient objects, machine-tool beds or cameras can be found in the literature (Asada and Granito, 1985; Gosselin and Angeles, 1989; Gosselin and Hamel, 1994; Di Gregorio, 2004); furthermore, 2-DOF wrists used as pointing devices have been proposed in Gosselin and Caron (1999) and Kong (2011). As described in Wu et al. (2014), two characteristic points can be identified in a spherical parallel wrist: the point at the intersection of the axes of the actuated joints at the base is the BP center, while the point at the intersection of the axes of the joints on the mobile platform is the MP center. Theoretically, the BP center and the MP center overlap with the spherical center. In reality, due to manufacturing errors and tolerances and to the deformation of the bodies under exter- nal loads, the joint axes do not intersect at a common point. If it is assumed that the base and the mobile platforms are perfectly rigid and manufactured with high-precision tools, we can still identify the BP and MP centers; however, they are shifted and the motion of the MP cannot be spherical.

The evaluation of the positioning error of a manipulator is a fundamental step in the design process if accuracy and stiffness are the guidelines for the mechanical project, as is the case for machining or assembly manipulators. Stiffness analysis of manipulators has been widely studied in recent years, mainly by means of analytic methods; examples include the studies of Majou et al. (2007), Pashkevich et al. (2009), Cammarata et al. (2012), Dong et al. (2014) and Wu et al. (2014). In most of these works of these works, the analytic model is exploited to evaluate the stiffness of the manipulator in a series of configurations inside the workspace. As the computational time of such models is quite low, a large number of configurations can be analyzed, obtaining, as a result, maps of the stiffness indices over the workspace of the machine. On the other hand, some simplifying assumptions are necessary to formulate the problem, and results may be affected by inaccuracies. The implementation of a finite-element model is usually done in order to validate the analytic model com- 
paring the results on a single or few configurations of the manipulator.

This paper investigates how the elastic deformation of the links and joints of a spherical parallel wrist generates an error in the positioning of the MP of the machine. Deformations are generated by an external load applied to the MP which simulates the interaction force exerted by an external manipulator during a cooperative assembly task. Recent advances in the field of flexible-multibody simulation tools today allow for a new approach to the stiffness analysis of manipulators to be pursued (Wu et al., 2014; Palmieri et al., 2014b): a configuration-dependent finite-element model, built as a result of searching for an optimum compromise between accuracy and computational effort, is directly used to perform the elasto-static analysis of the wrist in a series of configurations inside the workspace.

The remainder of the paper is organized as follows. Section 2 describes the kinematics and the mechanics of the parallel spherical wrist studied in this work; specifications and requirements of the machine are summarized and reference systems are fixed. After that, in Sect. 3, implementation of the finite-element model is discussed. Data obtained from the FEM analysis are then processed by means of a procedure, described in Sect. 4, which returns the positioning error of the MP in terms of displacement and orientation errors. Finally, results are discussed in Sect. 6.

\section{Kinematics and mechanical design of the spherical wrist}

The wrist studied in this paper is a non-overconstrained mini pointing device characterized by a parallel kinematics, which provides it with 2 DOFs of rotation. As described in Palmieri et al. (2014a), the mini wrist is conceived to be integrated in a mini assembly cell together with a second manipulator responsible for the Cartesian positioning; the final goal is to realize a fully automated mini cell for "general purpose" assembly tasks with accuracy and repeatability of a few microns and overall dimensions of a few centimeters. In this context, the emphasis given to the stiffness analysis and to the accuracy evaluation of the manipulators is fully motivated.

The functional and mechanical design of the device are described in Palmieri et al. (2015). The main characteristics of the wrist are a workspace of $\pm 54.7^{\circ}$ about every axis lying on the horizontal plane, a payload of $100 \mathrm{~g}$, an overall size contained within a cube with a side of $150 \mathrm{~mm}$, a circular MP with a $50 \mathrm{~mm}$ diameter, and a resolution on the order of $10^{-2 \circ}$. The kinematic structure of the wrist is based on the very common scheme of the parallel five-bar linkage (Gosselin and Caron, 1999; Kong, 2011). This well-known mechanism allows the platform to rotate with 2 DOFs around a point $\mathbf{O}$ located at the intersection of all the axes of the revolute joints. Starting from the basic scheme of the spherical five-bar linkage, in which all the joint axis are orthogonal,

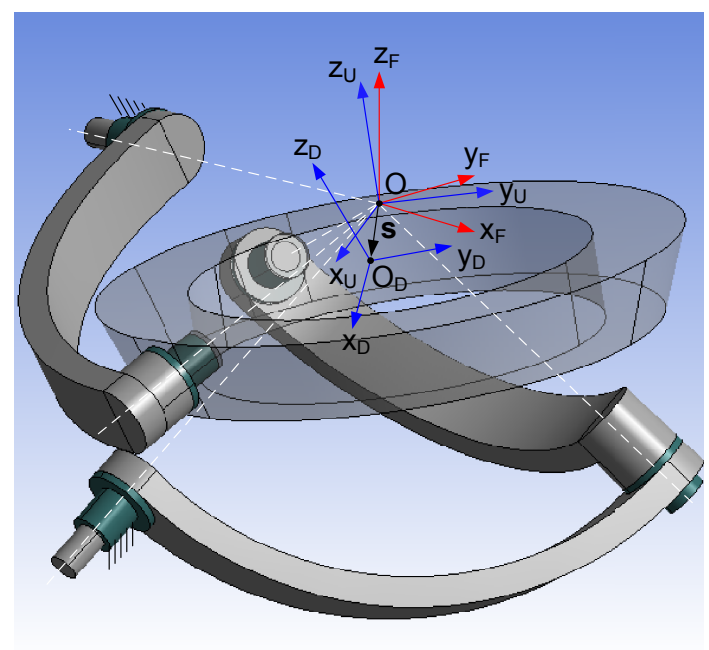

Figure 1. Frames attached to the BP $\{\mathcal{F}\}$, to the MP in the undeformed configuration $\{\mathcal{U}\}$ and to the MP in the deformed configuration $\{\mathcal{D}\}$.

a series of modifications have been introduced in order to guarantee the required workspace and to move the spherical center $\mathbf{O}$ of the wrist up from the supporting surface of the MP (Palmieri et al., 2015). The resulting kinematic scheme is shown in Fig. 1: in the hypothesis of rigid limbs, $\mathbf{O}$ is the intersection of all the joint axes and coincides with the BP center and with the MP center; the bushings marked with a hatched area are fixed to the frame and the rotations of the supported shafts are the motor angles. In the same figure, three different frames are introduced. The first frame, $\{\mathcal{F}\}$, is the frame of the BP with origin at the intersection of the axes of the two actuated joints. The frame associated with the MP in the undeformed configuration is $\{\mathcal{U}\}$, with the origin in common with $\{\mathcal{F}\}$ in $\mathbf{O}$; the relative rotation between $\{\mathcal{U}\}$ and $\{\mathcal{F}\}$ is due to the kinematics of the machine. When an external load is applied, the limbs are subjected to elastic deformations. As a result, the MP moves from its theoretical position to a new position identified by the frame $\{\mathcal{D}\}$. In this case, the center of the MP is shifted from $\mathbf{O}$ to $\mathbf{O}_{D}$ by a vector called $\boldsymbol{s}$, while the relative orientation between $\{\mathcal{U}\}$ and $\{\mathcal{D}\}$ is described by a rotation matrix called ${ }_{U}^{D} \mathbf{R}$.

The mechanical design of the wrist has been carried out until the realization of the prototype shown in Fig. 2. All of the links composing the limbs are made of steel, such as the frame, while the MP is made of aluminum alloy. An auxiliary plate and a pyramidal frame are shown in the figure, but they are not considered part of the wrist in the remainder of this study; in fact, such components constitute a particular design conceived for a demo task presented in Palmieri et al. (2015). According to Al-Widyan et al. (2011), all passive joints of the kinematic chain, which are ideally revolute joints, have been realized with bushings which also allow for small axial displacements; however in practice, they realize 


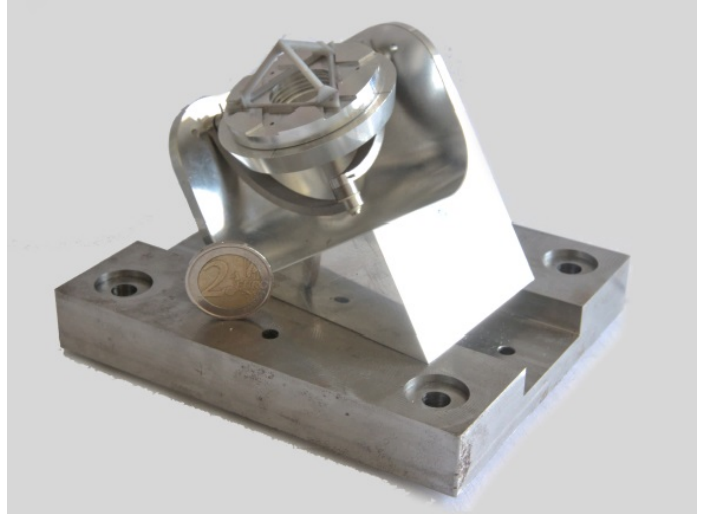

Figure 2. Prototype of the parallel spherical wrist.

cylindrical joints instead of revolute joints. With this expedient, the wrist behaves like a non-overconstrained mechanism that is able to cope with the manufacturing errors: machining tolerances lead to joint axes that inevitably do not intersect at a common point; thus the use of revolute joints would give a hyperstatic structure subjected to deformation when the links are assembled. On the other hand, the addition of extra DOFs in the passive joints introduces an undesired mobility which affects the accuracy and the stiffness of the machine. Due to the small dimensions of the wrist components and to the necessity of reduced friction, miniaturized polymeric bushings have been used; the low Young's modulus of such components introduces deformations concentrated at joints, which have been taken into account in the subsequent elasto-static analysis.

\section{Finite-element model}

The finite-element model was created in Ansys Workbench using the static structural module. A simplified CAD model of the wrist was imported, eliminating small features like chamfers and fillets and small components like screws and fasteners. The frame of the machine and the MP are considered perfectly rigid, while links and bushings at the joints are flexible. The material assigned to the links is steel (Young's modulus $E=200 \mathrm{GPa}$ ), while bushings are modeled in polymeric material with a Young's modulus of $2.4 \mathrm{GPa}$. The mesh is characterized by tetrahedral elements with refinements at the contact surfaces for a total of about 16000 elements and 28000 nodes (Fig. 3). The size of the elements was set by a sensitivity analysis: the detail level of the mesh was refined until results stabilized. Kinematic pairs have been introduced in order to replicate the isostatic kinematic structure of the wrist: all the bushings of the passive joints are fixed with the inner pin, while they are connected to the outer hub by a cylindrical pair; furthermore, frictionless surface contacts have been introduced between the flanges of the bushings and the corresponding surfaces of the connected bodies. Figure 4

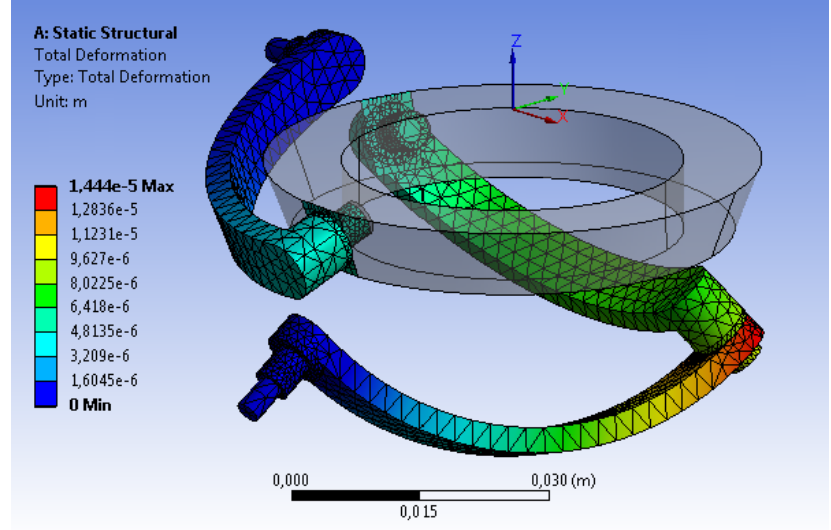

Figure 3. Mesh of the flexible components of the wrist and map of the total displacement for a load equal to $F=-1 \mathrm{~N}$ along the $z$ axes applied to the center of the MP.

shows the behavior of one of the modeled joints, where the translation of the cylindrical joint, in addition the rotation, is evident. The two bushings which support the pins connected to the motors are fixed to ground; each one of these bushings is connected to the corresponding pin by a revolute joint, and the relative corresponds to the motor rotation. By varying the motor angles, by means of the joint configuration tool, it is possible to configure the wrist in different positions in which the elasto-static analysis is then performed. A unitary vertical downward force is applied at the center of the MP. Such force simulates the interaction with an external manipulator during an assembly operation: the wrist orients an object that is aligning one of its surfaces with the horizontal plane, and the manipulator pushes against it in the normal direction. The magnitude of the force, set at $1 \mathrm{~N}$, corresponds to the payload of the wrist, equal to $100 \mathrm{~g}$. The deformation of the wrist is evaluated for each configuration by reading the displacement of seven points fixed to the MP; such data will be used in a subsequent procedure for the estimation of MP positioning error.

\section{Positioning error estimation}

Given a series of $n$ points fixed to the mobile platform, the position of the $i$ th point in the undeformed configuration with respect to the global fixed frame is called

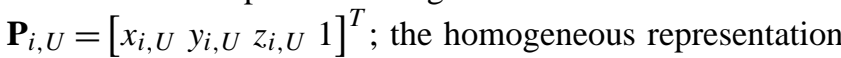
is used. After the load is applied, each one of those points moves according to the rigid body motion of the MP due to the elastic deformation of the limbs; its position in the deformed configuration is $\mathbf{P}_{i, D}=\left[\begin{array}{llll}x_{i, D} & y_{i, D} & z_{i, D} & 1\end{array}\right]^{T}$, again with respect to the global fixed frame. The following set of equations must be satisfied: 


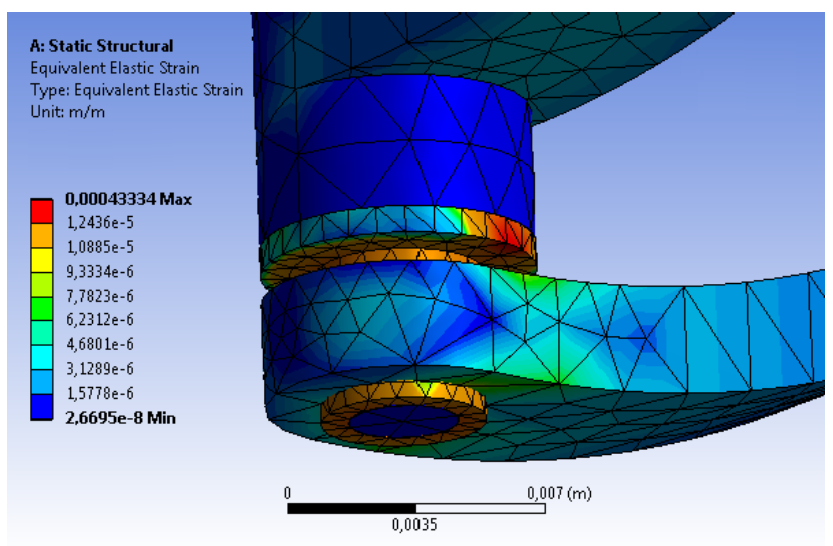

Figure 4. Modeling of the bushings: plot of the equivalent elastic strain on the deformed configuration (scale $100 \times$ ).

$\mathbf{P}_{i, D}={ }_{U}^{D} \mathbf{T P}_{i, U} \quad i=1,2, \ldots, n$.

${ }_{U}^{D} \mathbf{T}$ in Eq. (1) is the $4 \times 4$ homogeneous matrix representing the MP rigid motion of roto-translation due to the deformation of the limbs. As known, ${ }_{U}^{D} \mathbf{T}$ is composed of a rotation matrix ${ }_{U}^{D} \mathbf{R}$ and a vector $\boldsymbol{s}=\left(\mathbf{O}_{D}-\mathbf{O}\right)$, representing the relative orientation and the displacement between the origins of the undeformed $\{\mathcal{U}\}$ and the deformed $\{\mathcal{D}\}$ frames, respectively:

${ }_{U}^{D} \mathbf{T}=\left[\begin{array}{c|c}{ }_{U}^{D} \mathbf{R} & s \\ \hline \mathbf{0}^{T} & 1\end{array}\right]$

The coordinates of a series of $n$ points in the undeformed and deformed configuration are known from the results of the FEM analysis. They can be collected in the following matrices of dimension $4 \times n$ :

$\mathbf{A}_{U}=\left[\mathbf{P}_{1, U} \cdots \mathbf{P}_{n, U}\right] \quad \mathbf{A}_{D}=\left[\mathbf{P}_{1, D} \cdots \mathbf{P}_{n, D}\right]$.

Thus, on the basis of Eq. (1), a first estimation of the rototranslation matrix ${ }_{U}^{D} \mathbf{T}$ can be obtained as the result of an unconstrained least-squares problem:

${ }_{U}^{D} \widetilde{\mathbf{T}}=\mathbf{A}_{D} \mathbf{A}_{U}^{\dagger}$,

where ${ }^{\dagger}$ stands for the pseudo-inverse operator ${ }^{1}$ and $\sim$ is referred to first-estimate entities. Due to the numerical approximations of the FEM analysis, ${ }_{U}^{D} \widetilde{\mathbf{T}}$ does not match the constraints of a rigid body motion: its $3 \times 3$ submatrix ${ }_{U}^{D} \widetilde{\mathbf{R}}$, in particular, does not satisfy the orthogonality condition. A proper rotation matrix ${ }_{U}^{D} \mathbf{R}$ can be found as the orthogonal factor of the polar decomposition ${ }^{2}$ (Baron and Angeles,

\footnotetext{
${ }^{1}$ The Moore-Penrose pseudo-inverse of a $m \times n$ rectangular matrix A with $m<n$ can be directly calculated as $\mathbf{A}^{\dagger}=\mathbf{A}^{T}\left(\mathbf{A} \mathbf{A}^{T}\right)^{-1}$.

${ }^{2}$ The polar decomposition of a square matrix $\mathbf{A}$ is a matrix decomposition of the form $\mathbf{A}=\mathbf{Q W}$, where $\mathbf{Q}$ is an orthogonal matrix and $\mathbf{W}$ is a positive semi-definite Hermitian matrix.
}

2000; Higham, 1986) of the first unconstrained estimate ${ }_{U}^{D} \widetilde{\mathbf{R}}$ :

${ }_{U}^{D} \widetilde{\mathbf{R}}=\mathbf{Q} \mathbf{W}_{U}^{D} \mathbf{R}=\mathbf{Q}$.

Once ${ }_{U}^{D} \mathbf{R}$ is known, it is possible to rewrite the system of Eq. (1) in the form

$\mathbf{A}_{D}=\left[\begin{array}{c|c}{ }_{U}^{D} \mathbf{R} & \mathbf{0} \\ \hline \mathbf{0}^{T} & 1\end{array}\right] \mathbf{A}_{U}+\mathbf{S}$,

where

$\mathbf{S}=\left[\begin{array}{ccc}\boldsymbol{s}_{1} & \ldots & \boldsymbol{s}_{n} \\ 1 & & 1\end{array}\right]$.

Finally,

$\mathbf{S}=\mathbf{A}_{D}-\left[\begin{array}{c|c}{ }_{U}^{D} \mathbf{R} & \mathbf{0} \\ \hline \mathbf{0}^{T} & 1\end{array}\right] \mathbf{A}_{U}$

In the theoretical assumption of a rigid body motion, all vectors $s_{i}$ obtained from Eq. (8) are equal. In the actual case, they differ because of numerical approximations; thus, a least-squares problem has to be solved in order to find the translation vector $s$ that minimizes the error function $e$ :

$e=\frac{1}{n} \sum_{i=1}^{n}\left\|s-s_{i}\right\|^{2} \underset{s}{\rightarrow} \min$.

For data with a Gaussian noise distribution, it can be demonstrated (Lenarcic and Parenti-Castelli, 2001) that the result of the least-squares minimization is coincident with the mean value of the scattered samples. Thus, in our case, it is reasonable to assume that

$s=\frac{1}{n} \sum_{i=1}^{n} s_{i}$

The norm of $s$ represents the magnitude of the displacement of the MP center as a consequence of the elastic deformation. In order to also describe the orientation error by means of a scalar quantity, an axis-angle representation of the rotation matrix ${ }_{U}^{D} \mathbf{R}$ is used; the angle $\phi$ obtained from such a representation represents the magnitude of the orientation error of the MP.

The previously outlined procedure has been carried out for 25 different configurations of the wrist, which form an equally spaced grid over the joint space. Figures 5 and 6 show the plot of the displacement and orientation error, respectively, in the workspace of the wrist. Maps are obtained as interpolation of the data grid using biharmonic spline functions. A black solid line borders a subspace, called operative workspace, which represents the required range of motion for the particular application the wrist is designed for (Palmieri et al., 2015). The maximum shift $s$ of the MP center can reach up to $70 \times 10^{-3} \mathrm{~mm}$ at the borders of the workspace, while the maximum value of $\phi$ is of about $5.5 \times 10^{-2 \circ}$. Considering the data confined inside the operative workspace, 


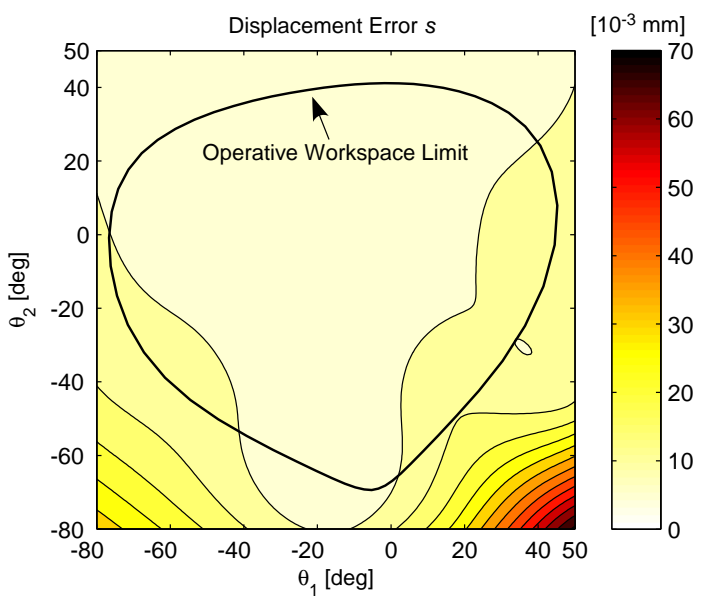

Figure 5. Displacement error $s$ of the MP mapped in the joint space.

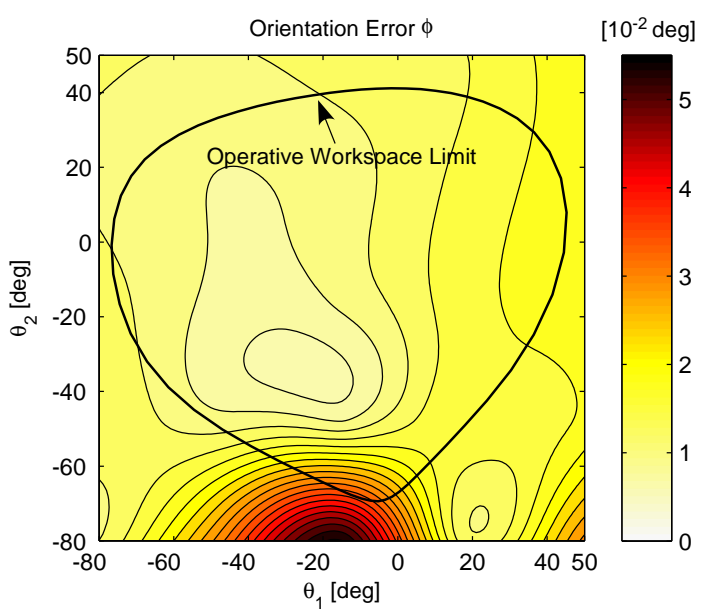

Figure 6. Orientation error $\phi$ of the MP mapped in the joint space.

the positioning errors are much lower: the mean, minimum and maximum values of $s$ and $\phi$ are shown in Table 1 ; the mean value of the orientation error $\left(1.29 \times 10^{-2 \circ}\right)$, in particular, is comparable with the angular resolution of the wrist $\left(1.5 \times 10^{-2 \circ}\right)$ related to the resolution of the encoders. The positions assumed by the center of the MP in the configurations analyzed inside the operative workspace generate a cloud of points whose convex hull is represented in Fig. 7. The location and the dimension of the hull can be evaluated on the basis of the statistical values of the components of the vector $s$ with respect to the frame $\{\mathcal{F}\}$, which are shown in Table 2.

\section{Conclusions}

The elasto-static analysis of a 2-DOF spherical parallel wrist where the links and the joints are considered flexible is described in this paper. An FEM approach has been proposed: starting from a simplified CAD of the manipulator, a re-

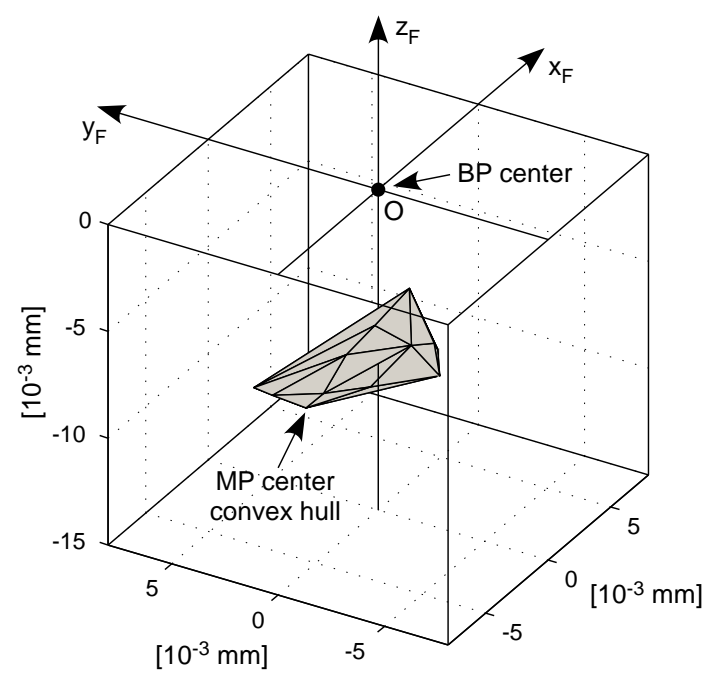

Figure 7. Convex hull of the positions of the MP center for a set of configurations of the wrist inside the operative workspace.

Table 1. Statistical values of the displacement error $s$ and orienting error $\phi$ inside the operative workspace.

\begin{tabular}{llccr}
\hline & & Mean & Min & Max \\
\hline$s$ & {$\left[10^{-3} \mathrm{~mm}\right]$} & 8.73 & 5.50 & 14.78 \\
$\phi$ & {$\left[10^{-2 \circ}\right]$} & 1.29 & 0.64 & 3.61 \\
\hline
\end{tabular}

Table 2. Statistical values of the components of the displacement error vector $s$ inside the operative workspace.

\begin{tabular}{rrrrr}
\hline & & Mean & Min & Max \\
\hline$s_{x}$ & {$\left[10^{-3} \mathrm{~mm}\right]$} & 1.17 & -2.06 & 5.12 \\
$s_{y}$ & {$\left[10^{-3} \mathrm{~mm}\right]$} & 0.53 & -2.12 & 5.63 \\
$s_{z}$ & {$\left[10^{-3} \mathrm{~mm}\right]$} & -8.39 & -13.51 & -5.11 \\
\hline
\end{tabular}

configurable finite-element model of the wrist has been implemented in Ansys Workbench. An external load is applied to the MP, simulating a cooperative assembly operation, and the consequent elastic deformation is evaluated. The displacements of a set of points fixed to the MP are then processed in a least-squares minimization procedure aimed to find the roto-translation that best fits the motion of the MP. Once the displacement and orientation errors are found for a series of configurations of the wrist, continuous maps over the workspace of the machine are obtained by interpolation. From examination of the operative workspace, it can be seen that the average orientation error is on the order of $10^{-2 \circ}$, which is compliant with the requirements of the wrist; moreover, the wrist exhibits a displacement error with an average value on the order of $10^{-2} \mathrm{~mm}$.

The main contribution of the work is the development of a novel methodology that allows for configuration-dependent 
stiffness analyses of manipulators with flexible limbs to be performed. The methodology is based on the interaction between multibody and FEM simulation tools and represents an alternative, or better an integration, to simplified analytic methods. Besides the evaluation of the positioning error, the proposed method can also be applied to perform an elastodynamic analysis of the wrist once a specific end-effector design is defined and mass properties of all components are known.

Acknowledgements. The present research was developed in the framework of the project PRIN2009 MM \& A, funded by the Italian Ministry for Education, Universities and Research (MiUR) and jointly carried out by the Polytechnic University of Marche, the University of Brescia, University of Bergamo and the Institute of Industrial Technologies and Automation of the Italian National Research Council.

Edited by: L. Birglen

Reviewed by: two anonymous referees

\section{References}

Al-Widyan, K., Ma, X. Q., and Angeles, J.: The robust design of parallel spherical robots, Mech. Mach. Theory, 46, 335-343, doi:10.1016/j.mechmachtheory.2010.11.002, 2011.

Asada, H. and Granito, J. A. C.: Kinematic and static characterization of wrist joints and their optimal design, in: vol. 2, IEEE T. Robot. Automat., 2, 244-250, 1985.

Baron, L. and Angeles, J.: The direct kinematics of parallel manipulators under joint-sensor redundancy, IEEE T. Robot. Automat., 16, 12-19, doi:10.1109/70.833183, 2000.

Cammarata, A., Condorelli, D., and Sinatra, R.: An Algorithm to Study the Elastodynamics of Parallel Kinematic Machines With Lower Kinematic Pairs, J. Mech. Robot., 5, 011004, doi:10.1115/1.4007705, 2012.

Di Gregorio, R.: The 3-RRS Wrist: A New, Simple and NonOverconstrained Spherical Parallel Manipulator, J. Mech. Design, 126, 850-855, doi:10.1115/1.1767819, 2004.

Dong, G., Song, Y., Sun, T. S., and Lian, B.: Elasto-dynamic Analysis of a Novel 2-DoF Rotational Parallel Mechanism, in: Proceedings of 2014 Workshop on Fundamental Issues and Future Research Directions for Parallel Mechanisms and Manipulators 7-8 July 2014, Tianjin, China, 2014.
Gosselin, C. and Angeles, J.: The Optimum Kinematic Design of a Spherical Three-Degree-of-Freedom Parallel Manipulator, J. Mech. Design, 111, 202-207, doi:10.1115/1.3258984, 1989.

Gosselin, C. and Caron, F.: Two degree-of-freedom spherical orienting device, United States patent US 5966991, 1999.

Gosselin, C. and Hamel, J.-F.: The agile eye: a highperformance three-degree-of-freedom camera-orienting device, IEEE T. Robot. Automat., 1, 781-786, doi:10.1109/ROBOT.1994.351393, 1994.

Higham, N.: Computing the Polar Decomposition - with Applications, SIAM J. Scient. Stat. Comput., 7, 1160-1174, doi:10.1137/0907079, 1986.

Kong, X.: Forward Displacement Analysis and Singularity Analysis of a Special 2-DOF 5R Spherical Parallel Manipulator, J. Mech. Robot., 3, 024501, doi:10.1115/1.4003445, 2011.

Lenarcic, J. and Parenti-Castelli, V.: A method for determining movements of a deformable body from spatial coordinates of markers, J. Robot. Syst., 18, 731-736, doi:10.1002/rob.8111, 2001.

Majou, F., Gosselin, C., Wenger, P., and Chablat, D.: Parametric stiffness analysis of the Orthoglide, Mech. Mach. Theory, 42, 296-311, doi:10.1016/j.mechmachtheory.2006.03.018, 2007.

Palmieri, G., Callegari, M., Carbonari, L., and Palpacelli, M.: Design and testing of a spherical parallel mini manipulator, in: IEEE/ASME Int. Conf. on Mechatronic and Embedded Systems and Applications, 10-12 September 2014, Senigallia, AN, Italy, 2014a.

Palmieri, G., Martarelli, M., Palpacelli, M., and Carbonari, L.: Configuration-dependent modal analysis of a Cartesian parallel kinematics manipulator: numerical modeling and experimental validation, Meccanica, 49, 961-972, doi:10.1007/s11012-0139842-4, 2014b.

Palmieri, G., Callegari, M., Carbonari, L., and Palpacelli, M.: Mechanical design of a mini pointing device for a robotic assembly cell, Meccanica, doi:10.1007/s11012-015-0132-1, in press, 2015.

Pashkevich, A., Chablat, D., and Wenger, P.: Stiffness analysis of overconstrained parallel manipulators, Mech. Mach. Theory, 44, 966- 982, doi:10.1016/j.mechmachtheory.2008.05.017, 2009.

Wu, G., Bai, S., and Kepler, J.: Mobile platform center shift in spherical parallel manipulators with flexible limbs, Mech. Mach. Theory, 75, 12-26, doi:10.1016/j.mechmachtheory.2014.01.001, 2014. 